\title{
Molecular and serological evidence of flea- associated typhus group and spotted fever group rickettsial infections in Madagascar
}

\author{
Rado J. L. Rakotonanahary ${ }^{1,2^{*}}$, Alan Harrison ${ }^{3}$, Alice N. Maina ${ }^{4}$, Ju Jiang ${ }^{4}$, Allen L. Richards ${ }^{4}$, \\ Minoarisoa Rajerison ${ }^{1}$ and Sandra Telfer ${ }^{3}$
}

\begin{abstract}
Background: Rickettsiae are obligate intracellular bacteria responsible for many febrile syndromes around the world, including in sub-Saharan Africa. Vectors of these pathogens include ticks, lice, mites and fleas. In order to assess exposure to flea-associated Rickettsia species in Madagascar, human and small mammal samples from an urban and a rural area, and their associated fleas were tested.

Results: Anti-typhus group (TGR)- and anti-spotted fever group rickettsiae (SFGR)-specific IgG were detected in 24 (39\%) and 21 (34\%) of 62 human serum samples, respectively, using indirect ELISAs, with six individuals seropositive for both. Only two (2\%) Rattus rattus out of 86 small mammals presented antibodies against TGR. Out of 117 fleas collected from small mammals, Rickettsia typhi, a TGR, was detected in 26 Xenopsylla cheopis (24\%) collected from rodents of an urban area $(n=107)$, while two of these urban X. cheopis (2\%) were positive for Rickettsia felis, a SFGR. $R$. felis DNA was also detected in eight (31\%) out of 26 Pulex irritans fleas.

Conclusions: The general population in Madagascar are exposed to rickettsiae, and two flea-associated Rickettsia pathogens, R. typhi and R. felis, are present near or in homes. Although our results are from a single district, they demonstrate that rickettsiae should be considered as potential agents of undifferentiated fever in Madagascar.
\end{abstract}

Keywords: Rickettsia, Rickettsioses, Fleas, Prevalence, Madagascar, Murine typhus, Flea-borne spotted fever

\section{Background}

Rickettsiae are Gram-negative obligate intracellular bacteria, closely associated with blood-feeding arthropods and subdivided in two groups: typhus group (TGR) and spotted-fever group (SFGR) [1]. They are responsible for many human infections resulting in mild to severe diseases, causing public health problems in many countries around the world. To our knowledge, there have been no confirmed reports of human cases of acute rickettsial infection from Madagascar. However, recent studies have revealed a high prevalence of Rickettsia africae, a SFGR, in Amblyomma ticks collected from cattle [2] and tortoises [3], as well as evidence of low rates of previous exposure to SFGR in pregnant women [2]. Most SFGR

* Correspondence: radoupty@pasteur.mg

${ }^{1}$ Plague Unit, Institut Pasteur de Madagascar, Antananarivo, Madagascar

${ }^{2}$ Ecole Doctorale Science de la Vie et de l'Environnement, Université

d'Antananarivo, Antananarivo, Madagascar

Full list of author information is available at the end of the article are tick-borne, however Rickettsia felis, the etiological agent of flea-borne spotted fever, is commonly associated with fleas [4]. Rickettsia typhi, a TGR, is another flea-borne Rickettsia and agent of murine typhus [5]. Although there is a lack of information concerning fleaborne Rickettsia in Madagascar, human cases of murine typhus and humans seropositive for anti-TGR antibodies have been reported in the neighbouring islands of Reunion and the Comoros archipelago, respectively $[6,7]$. The objectives of this study were to assess previous exposure of human populations from Madagascar to both SFGR and TGR, and to determine whether flea-borne rickettsiae circulate in peridomestic communities of small mammals and fleas.

\section{Methods}

The study was conducted in an urban area, Andrefanigara $\left(18^{\circ} 45^{\prime} 46^{\prime \prime} \mathrm{S}, 46^{\circ} 2^{\prime} 45^{\prime \prime} \mathrm{E}\right)$ and a rural area, Ambarivatry 
$\left(18^{\circ} 47^{\prime} 51^{\prime \prime} \mathrm{S}, 46^{\circ} 5^{\prime} 59^{\prime \prime} \mathrm{E}\right)$ within the Tsiroanomandidy district (Fig. 1) in January 2012. In each area, serum samples were collected from 31 healthy human participants. Selection of participants was conducted as follows. In each area, four departure points were randomly selected. For the urban area, the four departure points were located within an $800 \mathrm{~m}$ radius of the main health center, whilst for the rural area the four departure points were selected using a schematic diagram of the village overlaid with a grid. At each departure point, a direction of travel was randomly selected and houses then visited sequentially until 7-8 participants had been recruited. A maximum of three persons were recruited from a single household. All participants gave informed consent and were more than 18 years old (National ethical committee authorization 066-MSANP/CE).

To catch peridomestic small mammals, two traps [one wire-mesh trap (BTS) and one Sherman trap] were placed inside 15 and 20 houses in the rural and urban areas, respectively. Where possible a further BTS trap was set in the immediate vicinity of houses $(n=7$ in the urban area only). Further BTS traps were placed in outside trap-lines close to vegetation, for example adjacent to paths or areas used for growing crops or disposing of waste. Three lines with a total of 50 traps were set in the rural area and three lines with a total of 33 traps were set in the urban area. Thus, a total of 80 traps were set in both areas. Traps were baited with onion, dry fish and carrot and checked each morning for 3 days. Small mammals were identified by morphological characteristics, euthanized and blood samples taken via cardiac puncture. The fur was back-combed using a brush to collect fleas. The study was conducted in accordance with the Institut Pasteur (Paris) animal use guidelines (http://www.pasteur.fr/en/file/2626/download?token $=\mathrm{YgOq} 4 \mathrm{QW} 7$ ) and was approved by the local committee of the Institut Pasteur de Madagascar. Additional flea samples were trapped from 60 houses using light traps [8] during a plague investigation in another rural village within Tsiroanomandidy district, Miandrarivo (18 $56^{\prime} 39^{\prime \prime}$ S, 45 59'0"E) (Fig. 1) in October 2012. All blood samples were centrifuged and sera stored in liquid nitrogen before laboratory storage at $-80{ }^{\circ} \mathrm{C}$. Fleas were stored in $95 \%$ ethanol.

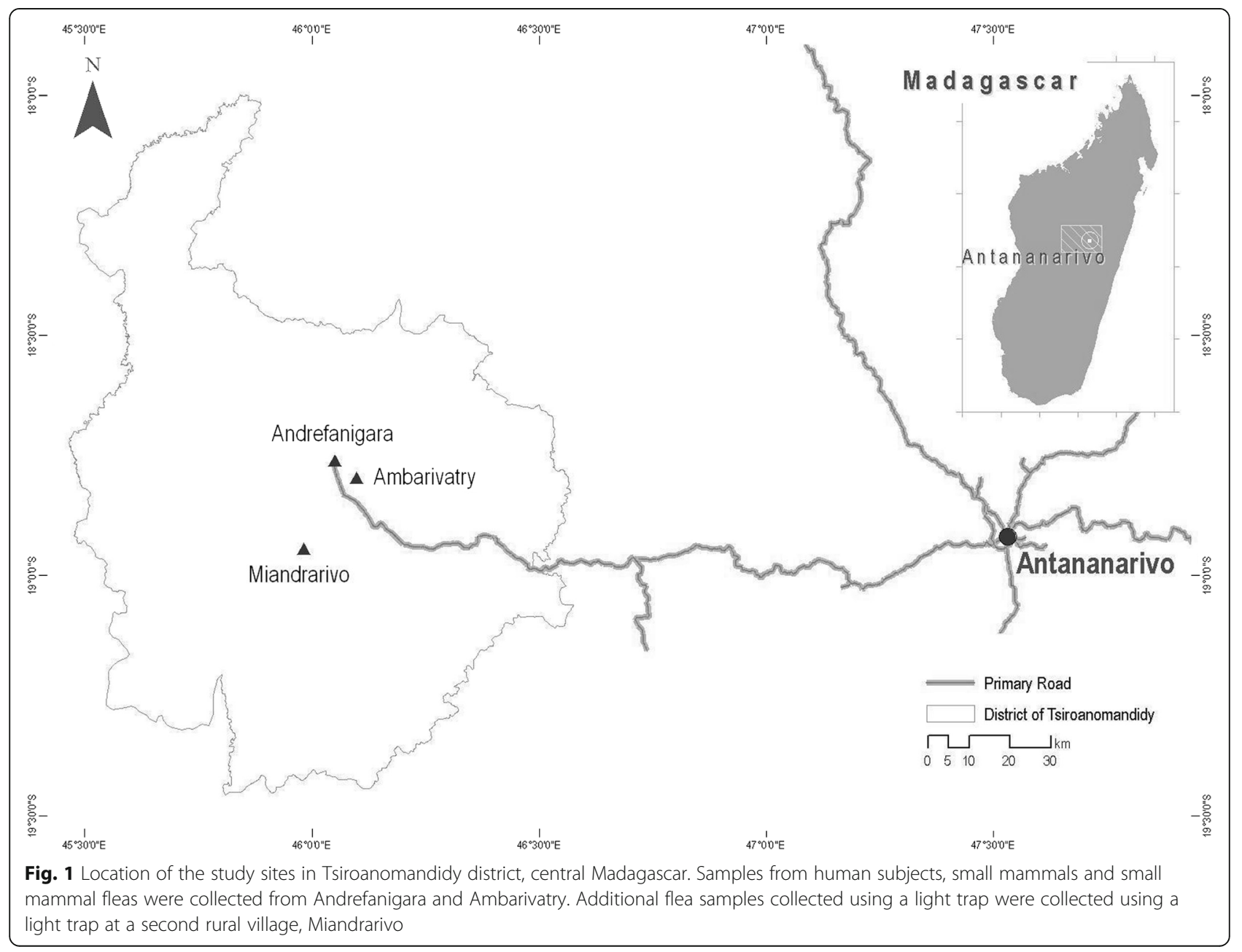


To assess previous exposure to Rickettsia spp., TGR- and SFGR-specific indirect IgG-ELISAs were conducted using human, rat and shrew sera. ELISAs were performed as described previously [9] with slight modifications. R. typhi Wilmington strain and Rickettsia conorii Malish strain whole-cell antigen preparations provided by the Naval Medical Research Center (NMRC), Maryland, USA, were used as TGR and SFGR ELISA antigen, respectively. Coating and blocking buffer was PBS-X powder (SIGMA, Saint Louis, MO) dissolved within ultra-pure water (Gibco, Life Technologies, Paisley, UK). Peroxidase labelled anti-human IgG (KPL, Gaithersburg, MD), antirat IgG peroxidase-conjugate (Sigma, Saint Louis, Missouri, USA) and protein-A peroxidase (Sigma) were used as secondary antibodies for human, rat and shrew sera, respectively. Secondary antibody dilutions were chosen to optimise the performance of the ELISAs according to the difference in absorbance (optical density, OD) of negative and positive controls. Anti-human secondary antibodies dilutions were 1:4000 for TGR and 1:3000 for SFGR ELISA, while anti-rat conjugate and protein-A conjugate were diluted at 1:2000. The absorbance was read at 405 nm using ELx800 spectrophotometer (Biotek, Winooski, VT) after $15 \mathrm{~min}$ of incubation. On each plate, one positive control and three negative controls were included. The positive controls for TGR and SFGR (human and mouse) were provided by NMRC. The human negative controls for TGR and SFGR were pools of Malagasy human sera confirmed negative for TGR and SFGR at the NMRC. The negative rodent controls were obtained from black rats born in captivity at Institut Pasteur de Madagascar. A sample was considered positive when the ratio (net $\mathrm{OD} /$ mean three negative controls + $3 S D) \geq 2$. Positive samples were subsequently included in a titration ELISA with four-fold dilution of the sera from $1: 100$ to $1: 6400$ to determine the individuals' antibody titer.

Flea species were identified using a dissecting microscope using morphological keys for all fleas described in Madagascar [10]. DNA was individually extracted using an in-house protocol. Briefly, fleas were dried, added to $150 \mu \mathrm{l}$ of brain-heart infusion broth, macerated with $5 \mathrm{~mm}$ steel beads using a Tissue-Lyser II (Qiagen, Hilden, Germany), and then centrifuged at $14,000 \mathrm{rpm}$ for $10 \mathrm{~s}$. The extraction then followed a previously described technique [11], with DNA dissolved in $60 \mu$ l of Tris-EDTA ( $\mathrm{pH} 8$ ). To detect DNA contamination, a negative control (water) was included for each 10 fleas.

All fleas collected from small mammals $(n=117)$ and one flea per house in Miandrarivo $(n=26)$ were screened for the presence of Rickettsia spp. using the genusspecific real-time PCR assay with RKND03F (5'-GTG AAT GAA AGA TTA CAC TAT TTA T-3'), RKND03R (5'-GTA TCT TAG CAA TCA TTC TAA TAG C-3') and RKND03P (5' -FAM-CT ATT ATG CTT GCG GCT GTC GGT TC-TAM RA-3'), based on the gltA gene [12]. Rickettsia-positive fleas were subsequently assessed using two real-time PCR assays targeting the ompB genes of $R$. typhi using RT557F (5'-TGG TAT TAC TGC TCA ACA AGC T-3'), RT678R (5'-CAG TAA AGT CTA TTG ATC CTA CAC C-3') and RT640BP (5'-FAM-CG CGA TCG TTA ATA GCA GCA CCA GCA TTA TCG CG-TAM RA-3'); and $R$. felis with RF1396F (5'-ACC CAG AAC TCG AAC TTT GGT G-3'), RF1524R (5'-CAC ACC CGC AGT ATT ACC GTT-3') and RF1448BP (5'-FAM-CG CGA CTT ACA GTT CCT GAT ACT AAG GTT CTT ACA GGT CGC G-T AMR A-3') [13]. Samples with a cycle threshold $(\mathrm{Ct})$ lower than 40 were considered positive. Thus, to be considered positive for TGR or SFGR, a sample had to have a Ct lower than 40 for both the genus-specific and species-specific assays. Each assay was run with negative and positive controls. Negative controls were distilled water and negative controls from DNA extraction. Positive controls were Rickettsia montanensis DNA for genus-specific qPCR, and plasmids containing target sequence of $R$. typhi and $R$. felis ompB fragment for species-specific qPCR assays.

To obtain amplicons suitable for sequencing, a subset of samples were chosen to be representative of results from the genus-specific and species-specific qPCR assays. These included Xenopsylla cheopis and Pulex irritans fleas and a range of $\mathrm{Ct}$ values. These samples were included in a semi nested-PCR assay targeting a fragment of $о \operatorname{mp}$. The first round PCR was run with primers 120-M59F (5'-CCG CAG GGT TGG TAA CTG C-3') and 1570R (5'-TCG CCG GTA ATT RTA GCA CT-3') [14, 15]. In the second round, 120-807R (5'-CCT TTT AGA TTA CCG CCT AA-3') [14] was paired with 120-M59F which amplify a fragment of approximately $833 \mathrm{bp}$ of $\mathrm{ompB}$ gene, and $1 \mu \mathrm{l}$ of first round PCR products were used as template. PCR products were visualized on 1.5\% agarose gel, purified with an Isolate PCR and Gel Kit (Bioline, London, UK) and sequenced in both directions using a commercial sequencing service (Eurofins, Ebersberg, Germany). Sequences were aligned and phylogenetic analyses performed using Mega 6.0 [16], analyses included $о m p B$ sequences from positive fleas and reference sequences from rickettsiae retrieved from GenBank (https://www.ncbi.nlm.nih.gov/ genbank/). The phylogenetic trees were constructed using maximum likelihood method and the bootstrap analyses were performed with 1000 replications.

Statistical analyses on serological and qPCR data were conducted using $\mathrm{R}$ software [17]. Associations were tested using Fisher exact tests. A P-value less than 0.05 was considered significant. 


\section{Results}

Fifty-two small mammals were captured from Andrefanigara (44 Rattus rattus; 6 Mus musculus; 2 Suncus muri$n u s)$ and 41 rodents from Ambarivatry ( $40 \mathrm{R}$. rattus; $1 \mathrm{M}$. musculus). From urban small mammals, 111 fleas were collected from 22 rodents, consisting of 107 Xenopsylla cheopis and 4 Synopsyllus fonquerniei. Rats from houses and their immediate vicinity were more often infested with $X$. cheopis $(87.5 \%, n=16)$ than those from outdoor lines around vegetation $(17.9 \%, n=28 ; P<0.001$, odds ratio $=28.62 ; 95 \%$ CI: 4.60-336.22). Six fleas were obtained from $3 R$. rattus trapped in Ambarivatry, including $1 X$. cheopis and 5 S. fonquerniei. In Miandrarivo, Pulex irritans were found in 26 (43.3\%) out of 60 houses.

Overall 39 and $34 \%$ of human serum samples tested positive for IgG against TGR and SFGR respectively (Table 1). There was no significant difference in prevalence between urban and rural areas for either TGR $(P=0.43)$ or SFGR $(P=0.59)$. There was also no evidence of an increase in seroprevalence with age for TGR [18-24 years $35 \%(n=12) ; 25-34$ years $29 \%(n=14)$; $35-49$ years $58 \%(n=12)$; > 49 years $38 \%(n=16)$; $P=0.49$ ] or SFGR (18-24 years $35 \%$, 25-34 years $36 \%$, $35-49$ years $8 \%,>49$ years $50 \% ; P=0.13)$. Evidence of a difference in seroprevalence for SFGR between males and females approached significance (males $44 \%(n=39)$, females $19 \%(n=23) ; P=0.052)$. No such pattern was observed for TGR (males 33\%, females $48 \%(n=23)$; $P=0.42$ ). Titers of anti-SFGR- and anti-TGR-specific IgG detected in human sera ranged from 100 to 6400. The geometric mean titers were 673 for antiTGR IgG and 635 for anti-SFGR antibodies.

Two rat samples from the 46 small mammals collected in the Andrefanigara were positive for anti-TGR antibodies based on the ratio method (Table 1). The titers of antibodies against TGR detected in the $2 R$. rattus were $>6400$. No rodent from Ambarivatry presented with antibodies to TGR, and anti-SFGR IgG were not detected in small mammal sera from either area.

Results of rickettsial DNA detection in fleas from Tsiroanomandidy are shown in Table 2. Of the X. cheopis fleas tested from 19 rats and one mouse caught in Andrefanigara $(n=107), 24 \%$ were positive for $R$. typhi DNA and $2 \%$ for $R$. felis DNA. Fleas positive for $R$. typhi DNA were collected from eight rats, and for these rats the proportion of positive fleas ranged from $7 \%(n=15$ fleas) to $100 \%$ (four rats, $n=1,6,2,3$ ), with a median of $90 \%$, indicating a very clumped distribution of infected fleas. Rats from houses and their immediate vicinity were not more likely to carry infected fleas $(42 \%, n=14)$ than those from outdoor lines around vegetation $(40 \%$, $n=5)$. Of the $P$. irritans fleas tested from Miandrarivo, $31 \%(n=26)$ were positive for $R$. felis DNA.

Rickettsia spp. ompB sequences were obtained from 6 $P$. irritans from Miandrarivo and $6 X$. cheopis from Andrefanigara (GenBank accession numbers KX090272KX090283). These sequencing data were consistent with results from the species-specific qPCR assays and revealed four variants of $R$. felis and a single variant of $R$. typhi (Fig. 2): $R$. felis from $2 X$. cheopis and 3 P. irritans were $99.4-100 \%$ identical to $R$. felis California 2, $R$. felis from $3 P$. irritans were $99.9-100 \%$ identical to $R$. felis clone Ar3 and $R$. typhi from 4 X. cheopis were 100\% identical to $R$. typhi strain Wilmington. The best DNA substitution model fitting the data was determined to be GTR + G. The maximum likelihood phylogenetic tree based on these fragments of $о т p B$ gene is shown in Fig. 2 .

\section{Discussion}

Rickettsial infections occur worldwide and may cause serious illness for humans. Recent studies in Madagascar focussed on tick-borne Rickettsiae [2, 3]. We reported here the first molecular evidence of $R$. typhi and $R$. felis in fleas from Madagascar. Rickettsia typhi was found in $X$. cheopis fleas from black rats, with rats in proximity to houses having higher flea infestations. Rickettsia felis was also closely associated with humans, being found predominantly in $P$. irritans. Consistent with our findings of peridomestic fleas infected with rickettsial pathogens from both groups, we found evidence within the human populations studied of previous exposure to both TGR and SFGR pathogens.

Table 1 Seroprevalence of antibodies against TGR and SFGR in humans and small mammals from Tsiroanomandidy, Madagascar. Exact binomial confidence intervals $(95 \% \mathrm{Cl})$ are given in square brackets

\begin{tabular}{lllll}
\hline Tested sera & Area & No. sampled & No. anti-TGR IgG positive (\%) [95\% Cl] & No. anti-SFGR IgG positive (\%) [95\% Cl] \\
\hline Human & Andrefanigara & 31 & $14(45.2)[27.8-63.7]$ & $9(29)[14.9-48.2]$ \\
& Ambarivatry & 31 & $10(32)[17.3-51.5]$ & $12(39)[22.4-57.7]$ \\
& Total & 62 & $24^{\mathrm{a}}(39)[26.9-52]$ & $21^{\mathrm{a}}(34)[22.6-47.1]$ \\
Rattus rattus $^{\mathrm{b}}$ & Andrefanigara & 44 & $2(4.5)[0.8-16.7]$ & 0 \\
& Ambarivatry & 40 & 0 & 0 \\
& Total & 84 & $2(2.4)[0.4-9.1]$ & 0 \\
\hline
\end{tabular}

${ }^{\text {a }}$ Six individuals were seropositive for both anti-TGR and anti-SFGR IgG

${ }^{\mathrm{b}}$ Sera samples from 2 Suncus murinus captured in Andrefanigara were also tested, both were negative 
Table 2 Detection of rickettsial DNA in fleas collected from rodents and houses from Tsiroanomandidy, Madagascar. Exact binomial confidence intervals $(95 \% \mathrm{Cl}$ ) are given in square brackets

\begin{tabular}{lllllll}
\hline Flea species & Principal host & Location & No. sampled & $\begin{array}{l}\text { Rickettsia spp. positive } \\
(\%)[95 \% \mathrm{Cl}]\end{array}$ & $\begin{array}{l}\text { R. typhi positive } \\
(\%)[95 \% \mathrm{Cl}]\end{array}$ & $\begin{array}{l}\text { R. felis positive } \\
(\%)[95 \% \mathrm{Cl}]\end{array}$ \\
\hline X. cheopis $^{\mathrm{a}}$ & Rodents & Andrefanigara & 107 & $36(33.6)[25-43.5]$ & $2^{26^{\mathrm{b}}(24.3)[16.8-33.7]}$ & $2^{\mathrm{b}}(1.9)[0.3-7.2]$ \\
P. irritans & Humans & Miandrarivo & 26 & $15(57.7)[37.2-76]$ & 0 & $8^{\mathrm{b}}(30.8)[15.1-51.9]$
\end{tabular}

${ }^{a}$ Other fleas collected from rodents were all negative: S. fonquerniei from Andrefanigara $(n=4)$ and Ambarivatry $(n=5)$ and $X$. cheopis from Ambarivatry ( $\left.n=1\right)$

${ }^{\mathrm{b}}$ Most Rickettsia spp. positive samples that were not subsequently positive for $R$. typhi or $R$. felis had Ct values close to 40

A previous study of apparently healthy participants in seven African countries that used an indirect immunofluorescent antibody assay (IFA) found seroprevalences of antibodies against $R$. conorii, a SFGR member, ranging from $0 \%$ in the Comoros to $46 \%$ in Angola, whilst seroprevalences of antibodies against $R$. typhi ranged from $0 \%$ in Angola to $11 \%$ in Mali [4]. Interestingly, some of the lowest prevalences were in the Comoros (TGR 2\%; SFGR $0 \%$ ), a group of islands close to Madagascar. A recent study, also using $R$. conorii as an SFGR antigen in an IFA test, and samples from pregnant women from six sites in
Madagascar, found a low seroprevalence of anti-SFGR antibodies (0.5-3.1\%) [2]. These results are compared with our anti-SFGR and anti-TGR IgG seroprevalences of 34 and 39\%, respectively. Thus, our results from an antiSFGR ELISA fall within the wide range of seroprevalences found in other African countries, but are much higher than the recent study in Madagascar. Interestingly, in our study we observed that females showed a lower seroprevalence for SFGR (19\%) than males (44\%). The low seroprevalence in the Keller et al. [2] study may therefore be related to the group sampled. Further work is needed to

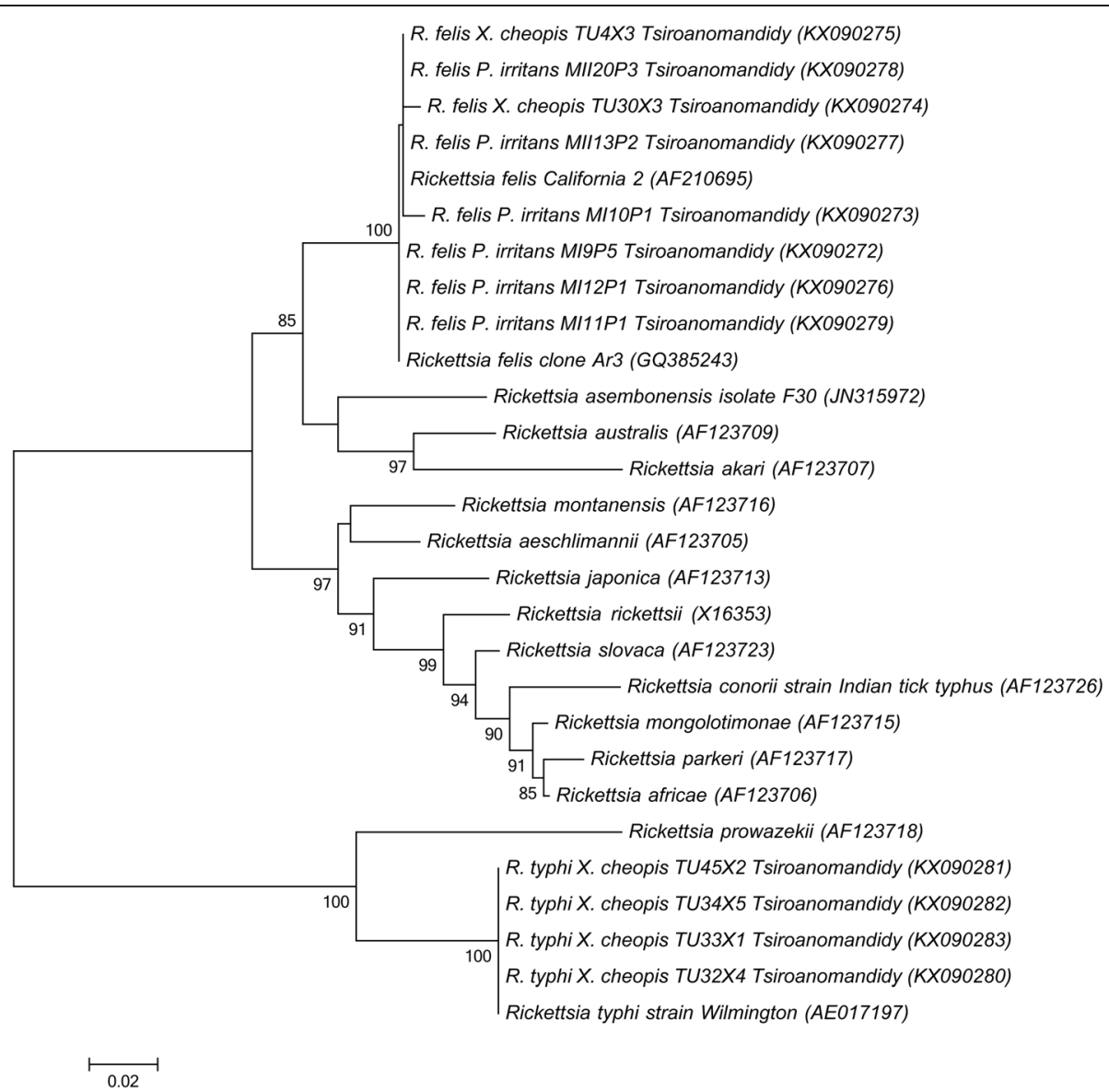

Fig. 2 Maximum likelihood phylogeny of Rickettsia species detected in fleas from Tsiroanomandidy based on ompB gene. Phylogenetic analysis was performed with $801 \mathrm{bp}$ and 795 fragment of $\mathrm{mpB}$ gene of $R$. felis and R. typhi, respectively, with GTR $+\mathrm{G}$ as the best DNA substitution model. Bootstrap values $>80 \%$ are presented. The scale bar on the bottom of the tree represents the number of substitutions per site. GenBank accession numbers are provided within brackets 
assess whether the prevalence of antibodies to SFGR differs between groups within the population, between sites or whether differences between studies are predominantly due to differing sensitivities of diagnostic tests. Compared with results from other African countries our TGR prevalence appears unusually high. With one of the highest incidences of plague in the world, the human population in Madagascar has a known exposure to diseases transmitted by rats and $X$. cheopis fleas, and Tsiroanomandidy district has a particularly high plague incidence [18]. Thus high exposure to $X$. cheopis fleas may explain the high exposure rate to TGR. A study in Malang, Indonesia, a city with a history of plague, reported similarly high anti-TGR IgG seroprevalences, with a significant increase from rural to suburban to urban population $(28,33$ and $42 \%$, respectively) [19]. We found no evidence of a significant difference in seroprevalence between urban and rural human populations in Tsiroanomandidy district, despite a much higher abundance of $X$. cheopis fleas in the urban site and a lack of evidence of infection in the few $X$. cheopis sampled at the rural site. Clearly, our flea data represented a single time point, whilst seroprevalence in the human population reflected exposure rates over time, and more data are needed to assess urban-rural differences in flea abundance, flea infection rates and anti-TGR IgG seroprevalence in the human population.

Anti-TGR and SFGR IgG were detected in six human sera. Thus $25 \%$ of samples that reacted to TGR antigens also reacted to SFGR antigens. This is a similar proportion to the findings reported in the Dupont et al. [7] study of apparently healthy participants (33\%) [4], but much lower than the proportion of dual-positive samples reported during a study on febrile patients from Kenya (90\%) [20]. Cross-reactivity between SFGR and TGR antigens had been reported [21, 22], and there had been suggestion that antibodies to $R$. felis may be a primary source of these cross-reactions [23]. However, more recently a study using the same ELISA approach as used here reported that antibodies directed against $R$. felis reacted with SFGR antigens, but not with TGR antigens [24]. Thus, our dual-positive sera may reflect previous exposure to both TGR and SFGR pathogens.

Whilst the group-specific ELISA tests are useful for an epidemiological serosurvey, the gold standard for diagnostics is IFA, followed by western blot, as this is more specific and can provide information on the most likely infecting Rickettsia sp. Apart from R. typhi, the TGR also includes $R$. prowazekii. As this louse-borne pathogen is most common in Africa in cooler, high altitude areas $[25,26]$ and our data indicated high prevalence of $R$. typhi in fleas, we believe exposure to $R$. typhi is more likely. A range of SFGR are known to occur in Africa, with most of these transmitted by ticks [27], and our SFGR results may, therefore, reflect an exposure to multiple SFGR pathogens. In the Dupont et al. [7] study, a subset of samples with a high titre to $R$. conorii antigens by IFA were used in a western blot against $R$. conorii and $R$. africae (another SFGR group member). The results indicated that the majority of sera reacted most strongly against $R$. africae [4]. Moreover, whereas the main ticks associated with transmission of $R$. conorii, Rhipicephalus spp., are found throughout Africa, the prevalence of antibodies to SFGR appeared highest in areas where Amblyomma ticks (commonly associated with cattle) are found. This led Dupont et al. [7] to speculate that much of the exposure to SFGR in subSahelian Africa may be due to $R$. africae rather than $R$. conorii. However, since this study, several studies have found that $R$. felis may be an important cause of febrile disease in Africa [12, 20, 28]. A recent study found a high prevalence of $R$. africae in Amblyomma ticks collected from cattle in Madagascar but, as described above, a low seroprevalence of anti-SFGR antibodies in pregnant women using an IFA test [2]. In the same study, a further IFA against $R$. africae antigen with strong positive samples from the original test indicated titres suggestive of previous $R$. africae infection. However, reactivity to $R$. felis antigens was not assessed. It was not, therefore, currently possible to ascertain the proportion of the population exposed to $R$. africae, $R$. felis or other SFGR species and further work is needed to confirm the identity of SFGR infecting the Malagasy population.

Awareness of rickettsioses amongst clinicians in Madagascar is low. Given the high seroprevalences of antibodies to Rickettsia spp. in the general population of Tsiroanomandidy district, rickettsioses may be underdiagnosed as causes of undifferentiated fever, as had been reported in studies of febrile patients in Kenya, Tanzania and Senegal $[12,20,29,30]$. These previous studies confirmed the potential of both $R$. typhi and $R$. felis to cause disease in humans. Similar studies are needed to determine the role of rickettsial agents in febrile syndromes in Madagascar.

Our results indicate that $X$. cheopis is likely to be a major vector for murine typhus in Madagascar, as has been found elsewhere [5]. Rickettsia felis is most commonly associated with the cat flea, Ctenocephalides felis $[4,31]$. However, it had also been found in other flea species, including $X$. cheopis [32-34]. A study in the Democratic Republic of Congo using an unspecified $R$. felis-specific qPCR reported $R$. felis in P. irritans [35]. More recently, in Senegal, although the same R. felisspecific qPCR assay as used in this study detected rickettsial DNA in P. irritans fleas, further testing revealed that this was actually detection of a new Rickettsia sp., Rickettsia asembonensis, which is closely related to $R$. felis but not known to cause disease in humans $[15,36]$. Our $R$. felis ompB sequence from $P$. irritans in 
Madagascar confirmed that this flea species can carry $R$. felis. However, the epidemiological cycle of $R$. felis in Africa remained unclear, with $R$. felis detected in a number of other arthropods, including mosquitoes and bed bugs [37].

Although we detected rickettsial DNA in X. cheopis collected from rats, seroprevalence of rat antibodies against Rickettsia spp. in this site was surprisingly low, with only two $R$. rattus out of 84 positive with anti-TGR IgG. One of the two seropositive rats carried no fleas, whilst the second carried $13 \mathrm{X}$. cheopis fleas, one of these fleas was positive for $R$. felis by qPCR. Our seroprevalence compares with high seroprevalence detected in several other studies. Antibodies to R. typhi were detected in $23.7 \%$ of rodents in Thailand [38], 11\% of Rattus captured in Jayapura, Indonesia [39] and 35.9\% of peridomestic rodents in Malang, Indonesia [19]. Our low seroprevalence may be due to a lack of sensitivity of our ELISA technique for rat sera, as we did not have a positive control that showed strong OD readings with the anti-rat conjugate for optimising the test (the mouse positive controls for both TGR and SFGR worked well with the protein-A peroxidase but consistently showed low OD readings with the anti-rat conjugate. Thus, we may have underestimated the number of rats with previous exposure to TGR. Although other mammals can act as reservoirs for murine typhus [30], it seems likely that $R$. rattus do play a role in Madagascar given their known reservoir capacity $[5,39]$ and the high percentage of $R$. typhi positive fleas feeding on them.

\section{Conclusion}

We found evidence here of exposure to both TGR and SFGR pathogens in the general human population, suggesting that rickettsioses should be considered as potential causes of undifferentiated fever in Madagascar. Molecular evidence demonstrates the presence of $R$. typhi and R. felis in fleas. We also confirm that the human flea, $P$. irritans, can be infected with $R$. felis, a Rickettsia species recently highlighted as an agent of febrile disease elsewhere in Africa. Further testing of human sera and flea samples from other districts is currently being conducted, and will provide more detail on the prevalence, distribution and risk of Rickettsia infections.

\section{Acknowledgements}

We would like to thank Jean-Michel Heraud, Marie-Marie Olive, Fehivola Andriamiarimanana and Corinne Rahaingosoamamitiana (all from Institut Pasteur de Madagascar) for helping to organize and conduct field work. We are grateful to Didier Raoult and Christina Socolovschi (Unité de Recherche sur les Maladies Infectieuses Tropicales Emergentes, Marseille, France) who kindly provided us DNA extracts of $R$. montanensis and $R$. prowazekii as qPCR positive controls.

\section{Funding}

This research was supported by the Wellcome Trust (RCDF and Senior Fellowship to ST, \#081705 and \#095171), the Institut Pasteur de Madagascar, and the Global Emerging Infections Surveillance and Response System, a Division of the Armed Forces Health Surveillance Center [847705.82000.25GB.A0074].

\section{Availability of data and materials}

DNA sequences generated and analyse during the current study are available in GenBank repository under accession numbers KX090272-KX090283.

\section{Authors' contributions}

MR and ST designed the project. RLJR, with help from AH, carried out the laboratory work, data analysis and wrote the manuscript. All authors were involved in intellectual interpretation and critical revision of the manuscript for publication. All authors read and approved the final manuscript.

\section{Competing interests}

The authors declare that they have no competing interests.

\section{Consent for publication}

Not applicable.

Ethics approval and consent to participate

Study on human subjects was approved by the national ethical committee under permit number 066-MSANP/CE, July 26th, 2011. All human participants gave informed consent to participate in the study and were more than 18 years old. The study using small mammals was conducted in accordance with the Institut Pasteur (Paris) guidelines (http://www.pasteur.fr/en/file/ 2626/download?token=YgOq4QW7) for animal husbandry and experiments, and was approved by the local "Ad-Hoc" committee of the Institut Pasteur de Madagascar, which includes members from the Science Faculty and Veterinarian School at the University of Antananarivo, and the Madagascar Ministry of Livestock.

\section{Disclaimer}

The views expressed in this article are those of the authors and do not necessarily reflect the official policy or position of the Department of the Navy, Department of Defense, nor the U.S. Government.

\section{Copyright statement}

ALR is an employee of the U.S. Government and this work was prepared as part of his official duties. Title 17 U.S.C. \$105 provides that 'Copyright protection under this title is not available for any work of the United States Government.' Title 17 U.S.C. \$101 defines a U.S. Government work as a work prepared by a military service member or employee of the U.S. Government as part of that person's official duties.

\section{Author details}

${ }^{1}$ Plague Unit, Institut Pasteur de Madagascar, Antananarivo, Madagascar. ${ }^{2}$ Ecole Doctorale Science de la Vie et de l'Environnement, Université d'Antananarivo, Antananarivo, Madagascar. ${ }^{3}$ Institute of Biological and Environmental Sciences, University of Aberdeen, Aberdeen, UK. ${ }^{4}$ Viral and Rickettsial Diseases Department, Naval Medical Research Center, Silver Spring, $M D$, USA.

Received: 4 November 2016 Accepted: 24 February 2017

Published online: 04 March 2017

\section{References}

1. Renvoisé A, Raoult D. L'actualité des rickettsioses. Med Mal Infect. 2009;39: 71-81.

2. Keller C, Krüger A, Schwarz NG, Rakotozandrindrainy R, Rakotondrainiarivelo JP, Razafindrabe T, et al. High detection rate of Rickettsia africae in Amblyomma variegatum but low prevalence of anti-rickettsial antibodies in healthy pregnant women in Madagascar. Ticks Tick Borne Dis. 2016;7:60-5.

3. Ehlers J, Ganzhorn JU, Silaghi C, Krüger A, Pothmann D, Ratovonamana RY, et al. Tick (Amblyomma chabaudi) infestation of endemic tortoises in southwest Madagascar and investigation of tick-borne pathogens. Ticks Tick Borne Dis. 2016;7:378-83.

4. Azad AF, Radulovic S, Higgins JA, Noden BH, Troyer JM. Flea-borne rickettsioses: ecologic considerations. Emerg Infect Dis. 1997;3:319-27.

5. Azad AF. Epidemiology of murine typhus. Annu Rev Entomol. 1990;35:553-69.

6. Balleydier E, Camuset G, Socolovschi C, Moiton M, Kuli B, Foucher A, et al. Murine typhus, Reunion, France, 2011-2013. Emerg Infect Dis. 2015;21:316-9.

7. Dupont HT, Brouqui P, Faugere B, Raoult D. Prevalence of antibodies to Coxiella burnetti, Rickettsia conorii, and Rickettsia typhi in seven African countries. Clin Infect Dis. 1995;21:1126-33. 
8. Ratovonjato J, Randriambelosoa J, Robert V. Tunga penetrans (Insecta, Siphonaptera, Tungidae) à Madagascar: une nuisance négligée. Rev Med Vet. 2008;159:551-6.

9. Graf PCF, Chretien J-P, Ung L, Gaydos JC, Richards AL. Prevalence of seropositivity to spotted fever group Rickettsiae and Anaplasma phagocytophilum in a large, demographically diverse US sample. Clin Infect Dis. 2008;46:70-1.

10. Duchemin J-B. Biogéographie des puces de Madagascar. [Thèse de doctorat]: Université de Paris XII-Val de Marne; 2003.

11. Cornel AJ, Collins FH. PCR of the ribosomal DNA intergenic spacer regions as a method for identifying mosquitoes in the Anopheles gambiae complex. Methods Mol Biol. 1996;50:321-32.

12. Socolovschi C, Mediannikov O, Sokhna C, Tall A, Diatta G, Bassene H, et al. Rickettsia felis-associated uneruptive fever, Senegal. Emerg Infect Dis. 2010;16:1140-2.

13. Henry KM, Jiang J, Rozmajzl PJ, Azad AF, Macaluso KR, Richards AL. Development of quantitative real-time PCR assays to detect Rickettsia typhi and Rickettsia felis, the causative agents of murine typhus and flea-borne spotted fever. Mol Cell Probes. 2007;21:17-23.

14. Roux V, Raoult D. Phylogenetic analysis of members of the genus Rickettsia using the gene encoding the outer-membrane protein rOmpB (ompB). Int J Syst Evol Microbiol. 2000;50:1449-55.

15. Jiang J, Maina AN, Knobel DL, Cleaveland S, Laudisoit A, Wamburu K, et al. Molecular detection of Rickettsia felis and Candidatus Rickettsia asemboensis in fleas from human habitats, Asembo, Kenya. Vector Borne Zoonotic Dis. 2013;13:550-8

16. Tamura K, Stecher G, Peterson D, Filipski A, Kumar S. MEGA6: Molecular Evolutionary Genetics Analysis version 6.0. Mol Biol Evol. 2013;30:2725-9.

17. R Core Team. R: A language and environment for statistical computing. Vienna: R Fund Stat Comput; 2014.

18. Andrianaivoarimanana V, Kreppel K, Elissa N, Duplantier JM, Carniel E, Rajerison $\mathrm{M}$, et al. Understanding the persistence of plague foci in Madagascar. PLoS Negl Trop Dis. 2013;7:1-8.

19. Richards AL, Soeatmadji DW, Widodo MA, Sardjono TW, Yanuwiadi B, Hernowati TE, et al. Seroepidemiologic evidence for murine and scrub typhus in Malang, Indonesia. Am J Trop Med Hyg. 1997;57:91-5.

20. Maina AN, Knobel DL, Jiang J, Halliday J, Feikin DR, Cleaveland S, et al. Rickettsia felis infection in febrile patients, Western Kenya, 2007-2010. Emerg Infect Dis. 2012:18:328-31.

21. Ormsbee R, Peacock M, Philip R, Casper E, Plorde J, Gabre-Kidan T, et al. Antigenic relationship between the typhus and spotted fever group of Rickettsiae. Am J Epidemiol. 1978;108:53-9.

22. Hechemy KE, Raoult D, Fox J, Han Y, Elliott LB, Rawlings J. Cross-reaction of immune sera from patients with rickettsial diseases. J Med Microbiol. 1989; 29:199-202.

23. Znazen A, Rolain J-L, Hammami N, Hammami A, Jemaa MB, Raoult D. Rickettsia felis infection, Tunisia. Emerg Infect Dis. 2006;12:138-40.

24. Maina AN, Fogarty C, Krueger L, Macaluso KR, Odhiambo A, Nguyen K, et al. Rickettsial infections among Ctenocephalides felis and host animals during a flea-borne rickettsioses outbreak in Orange County, California. PLoS One. 2016;11:e0160604

25. Mokrani K, Fournier PE, Dalichaouche M, Tebbal S, Aouati A, Raoult D. Reemerging threat of epidemic typhus in Algeria. J Clin Microbiol. 2004;42: 3898-900.

26. Perine PL, Chandler BP, Krause DK, McCardle P, Awoke S, Habte-Gabr E, et al. A clinico-epidemiological study of epidemic typhus in Africa. Clin Infect Dis. 1992;14:1149-58.

27. Berrelha J, Briolant S, Muller F, Rolain J-M, Marie J-L, Pagés F, et al. Rickettsia felis and Rickettsia massiliae in Ivory Coast, Africa. Clin Microbiol Infect. 2009; 15:251-2.

28. Parola P. Rickettsia felis: from a rare disease in the USA to a common cause of fever in sub-Saharan Africa. Clin Microbiol Infect. 2011;17:996-1000.

29. Crump JA, Morrissey AB, Nicholson WL, Massung RF, Stoddard RA, Galloway $\mathrm{RL}$, et al. Etiology of severe non-malaria febrile illness in northern Tanzania: a prospective cohort study. PLoS Negl Trop Dis. 2013;7:e2324.

30. Richards AL, Jiang J, Omulo S, Dare R, Abdirahman K, Ali A, et al. Human infection with Rickettsia felis, Kenya. Emerg Infect Dis. 2010;16:1081-6.

31. Reif KE, Macaluso KR. Ecology of Rickettsia felis: a review. J Med Entomol. 2009:46:723-36.

32. Eremeeva ME, Warashina WR, Sturgeon MM, Buchholz AE, Olmsted GK, Park SY, et al. Rickettsia typhi and R. felis in rat fleas (Xenopsylla cheopis), Oahu, Hawaii. Emerg Infect Dis. 2008;14:1613-5.
33. Jiang J, Soeatmadji DW, Henry KM, Ratiwayanto S, Bangs MJ, Richards AL. Rickettsia felis in Xenopsylla cheopis, Java, Indonesia. Emerg Infect Dis. 2006: 12:1281-3.

34. Christou C, Psaroulaki A, Antoniou M, Toumazos P, loannou I, Mazeris A, et al. Rickettsia typhi and Rickettsia felis in Xenopsylla cheopis and Leptopsylla segnis parasitizing rats in Cyprus. Am J Trop Med Hyg. 2010;83:1301-4.

35. Sackal C, Laudisoit A, Kosoy M, Massung R, Eremeeva ME, Karpathy SE, et al. Bartonella spp. and Rickettsia felis in fleas, Democratic Republic of Congo. Emerg Infect Dis. 2008;14:1972-4.

36. Maina AN, Luce-Fedrow A, Omulo S, Hang J, Chan T-C, Ade F, et al. Isolation and characterization of a new Rickettsia species (Rickettsia asembonensis sp. nov) obtained from cat fleas (Ctenocephalides felis). Int I Syst Evol Microbiol. 2016:66:4512-7.

37. Mediannikov O, Socolovschi C, Edouard S, Fenollar F, Mouffok N, Bassene H, et al. Common epidemiology of Rickettsia felis infection and malaria, Africa. Emerg Infect Dis. 2013;19:1775-83.

38. Chareonviriyaphap T, Leepitakrat W, Lerdthusnee K, Chao CC, Ching WM. Dual exposure of Rickettsia typhi and Orientia tsutsugamushi in the fieldcollected Rattus rodents from Thailand. J Vector Ecol. 2014;39:182-9.

39. Richards AL, Rahardjo E, Rusjdi AF, Kelly DJ, Dasch GA, Church CJ, et al. Evidence of Rickettsia typhi and the potential for murine typhus in Jayapura, Irian Jaya, Indonesia. Am J Trop Med Hyg. 2002;66:431-4.

\section{Submit your next manuscript to BioMed Central and we will help you at every step:}

- We accept pre-submission inquiries

- Our selector tool helps you to find the most relevant journal

- We provide round the clock customer support

- Convenient online submission

- Thorough peer review

- Inclusion in PubMed and all major indexing services

- Maximum visibility for your research

Submit your manuscript at www.biomedcentral.com/submit
) Biomed Central 